\title{
Constructing a solution in time semidiscretization method to an equation of vibrating string with an obstacle
}

\author{
Koji Kikuchi \\ Department of Applied Mathematics, Faculty of Engineering, Shizuoka University, \\ Hamamatsu 432-8561, Japan
}

\begin{abstract}
This article treats a problem which describs movement of a string that hits to an obstacle. In [10] M. Schatzman solves this problem in a slightly classical way. In [5], [6] K. Maruo constructs a solution to this problem by the use of Yosida approximation. The purpose of this article is to construct a solution to this problem in time semidiscretization method. In general approximation by time semidiscretization method is different from Yosida approximation. A simple example is presented in Appendix.
\end{abstract}

Key words: obstacle, a second order hyperbolic differential inequality, time semidiscretization

2000 MSC: 35L70, 35A15

\section{Introduction}

In [9] M. Schatzman treats a problem which describs movement of a string that hits to an obstacle. This problem is formulated as in the following way. Here, for the sake of simplicity, we suppose that the obstacle is flat just like as a table. Let $u_{0} \in W^{1,2}(0,1)$ and $v_{0} \in L^{2}(0,1)$ with $u_{0} \geq 0$ and $u_{0}(0)=u_{0}(1)=1$, and find $u$ that satisfies a second order hyperbolic differential inequality

$$
u_{t t}-u_{x x} \geq 0
$$

Email address: tskkiku@ipc.shizuoka.ac.jp (Koji Kikuchi). 
in the sense of distributions and

$$
\begin{aligned}
& \text { spt }\left(u_{t t}-u_{x x}\right) \subset\{u=0\} \\
& u(t, x) \geq 0 \quad \text { for } \quad \mathcal{L}^{2}-\text { a.e. }
\end{aligned}
$$

with initial conditions

$$
u(0, x)=u_{0}, \quad \frac{\partial u}{\partial t}(0, x)=v_{0}
$$

and a boundary condition

$$
u(t, 0)=u(t, 1)=1 \text {. }
$$

A weak solution to (1.1)-(1.5) is defined as follows:

Definition 1.1 A function $u:(0, T) \rightarrow L^{2}(0,1)$ is said to be a weak solution to $(1.1)-(1.5)$ in $(0, T)$ if

i) $u \in W^{1,2}((0, T) \times(0,1)), \quad u(t, x) \geq 0$ for $\mathcal{L}^{2}$-a.e. $(t, x)$

ii) $\mathrm{s}-\lim _{t \searrow 0} u(t)=u_{0}$ in $L^{2}(0,1)$

iii) $u(t, 0)=u(t, 1)=1$

iv) for any $\phi \in C_{0}^{0}\left([0, T) ; L^{2}(0,1)\right) \cap W_{0}^{1,2}((0, T) \times(0,1))$ with $\phi \geq 0$,

$$
-\int_{0}^{T} \int_{0}^{1} u_{t}(t) \phi_{t}(t) d x d t+\int_{0}^{T} \int_{0}^{1} u_{x} \phi_{x} d x d t-\int_{0}^{1} v_{0} \phi d x \geq 0 .
$$

v) for any $\phi \in C_{0}^{0}\left([0, T) ; L^{2}(0,1)\right) \cap W_{0}^{1,2}((0, T) \times(0,1))$ with spt $\phi \subset(\{u=$ $0\})^{c}$,

$$
-\int_{0}^{T} \int_{0}^{1} u_{t}(t) \phi_{t}(t) d x d t+\int_{0}^{T} \int_{0}^{1} u_{x} \phi_{x} d x d t-\int_{0}^{1} v_{0} \phi d x=0 .
$$

Replacing (1.3) with $u \geq r$, where $r \in C^{0}([0,1])$, we can treat a more complicated obstacle. Probably the case that $u(t, 0)=a, u(t, 1)=b$ with $a, b \geq 0$ is also a problem. Our analysis is available for these cases under some minor changes. 
In [10] M. Schatzman solves this equation in a slightly classical way. Under a condition that a solution satisfies an equality which assures the energy conservation law, uniqueness is also proved. In [5], [6] K. Maruo constructs a solution to this problem by the use of Yosida approximation. Let us define a functional $\Phi: L^{2}(0,1) \rightarrow[0, \infty]$ by

$$
\Phi(u)=\left\{\begin{array}{l}
0 \text { if } u(x) \geq 0 \text { for each } x \\
\infty \text { if otherwise }
\end{array}\right.
$$

Then (1.1)-(1.3) are equivalent to

$$
u_{t t}-u_{x x}+\partial \Phi(u) \ni 0
$$

in $L^{2}(0,1)$. In [5], [6] Yosida approximation is carried out to the functional $\Phi$, namely, given $\lambda>0$, approximate functional $\Phi_{\lambda}$ is defined as

$$
\Phi_{\lambda}(u)=\min \left\{\frac{1}{2 \lambda}\|v-u\|^{2}+\int_{0}^{1}\left|v_{x}(x)\right|^{2} d x+\Phi(v) ; v \in L^{2}(0,1)\right\} .
$$

Then $\partial \Phi_{\lambda}$ is singleton and Lipschitz continuous in $L^{2}(\Omega)$.

The purpose of this article is to construct a solution to this problem in the method of semidiscretization in time variable. This approximating method is often called Rothe's method and at first introduced to construct weak solutions to parabolic equations ([8]). In [11] it is pointed out that this method is also available for hyperbolic equations and semilinear hyperbolic equations are solved by the use of this method. In [2], [3] this method is applied to quasilinear hyperbolic equations, however in these works it should be supposed that the limit of approximate solutions satisfies energy conservation law. Although our equation has stronger nonlinearity than a semilinear equation, we obtain that passing to a subsequence if necessary approximate solutions converge to a solution without assuming any additional assumptions, such as energy conservation law. In time semidiscretization method we should solve elliptic equations with respect to space variables, and when the equation has divergence form, a direct variational method is one way of solving an elliptic equation; indeed in [2], [3], [11] elliptic equations are solved by minimizing variational functionals. In this respect this method is closely related to the theory of minimizing movements, which is proposed by E. De Giorgi [1]. In [12] the method of combining Rothe's time semidiscretization and minimizing functionals is refered to as minimizing movement method.

Now we overview the time semidiscretization method. For a positive number $h$ we construct a sequence $\left\{u_{l}\right\}_{l=-1}^{\infty}$ in the following way. For $l=0$ we let $u_{0}$ 
be as above and for $l=-1$ we set $u_{-1}=u_{0}-h v_{0}$. For $l \geq 1, u_{l}$ is defined as a solution to the time semidiscretized problem, which is equivalent to

$$
\frac{u-2 u_{l-1}+u_{l-2}}{h^{2}}-u_{x x}+\partial \Phi(u) \ni 0
$$

with (1.4), (1.5), where $\Phi$ is as in (1.6), and it is obtained as a minimizer of the functional

$$
\mathcal{F}_{l}(u)=\frac{1}{2 h^{2}}\left\|u-2 u_{l-1}+u_{l-2}\right\|^{2}+\int_{0}^{1}\left|u_{x}\right|^{2} d x+\Phi(u)
$$

in $W^{1,2}(0,1)$ with $u(0)=u(1)=1$. The existence of the minimizer is assured by lower semicontinuity and boundedness from below of $\mathcal{F}_{l}$. By the use of convexity of $\int_{0}^{1}\left|u_{x}\right|^{2} d x+\Phi(u)$ we have energy inequality

$$
\frac{1}{2 h^{2}}\left\|u_{l}-u_{l-1}\right\|^{2}+\int_{0}^{1}\left|\left(u_{l}\right)_{x}\right|^{2} d x+\Phi\left(u_{l}\right) \leq \frac{1}{2}\left\|v_{0}\right\|^{2}+\int_{0}^{1}\left|\left(u_{0}\right)_{x}\right|^{2} d x .
$$

(compare to [7]). Note that the right hand side is in fact $\frac{1}{2}\left\|v_{0}\right\|^{2}+\int_{0}^{1}\left|\left(u_{0}\right)_{x}\right|^{2} d x+$ $\Phi\left(u_{0}\right)$, however by the assumption on $u_{0}$ we have $\Phi\left(u_{0}\right)=0$.

Next we define approximate solutions $u^{h}(t)$ and $\bar{u}^{h}(t)$ for $t \in(-h, \infty)$ as follows: for $(l-1) h<t \leq l h$

$$
u^{h}(t, x)=\frac{t-(l-1) h}{h} u_{l}(x)+\frac{l h-t}{h} u_{l-1}(x)
$$

and

$$
\bar{u}^{h}(t, x)=u_{l}(x) .
$$

Then we have by $(1.7)$

$$
\frac{1}{2} \int_{0}^{1}\left|u_{t}^{h}(t)\right|^{2} d x+\int_{0}^{1}\left|\bar{u}_{x}(t)\right|^{2} d x+\Phi\left(\bar{u}^{h}(t)\right) \leq \frac{1}{2} \int_{0}^{1}\left|v_{0}\right|^{2} d x+\int_{0}^{1}\left|\left(u_{0}\right)_{x}\right|^{2} d x(1.10)
$$


for each $t \in \bigcup_{l=0}^{\infty}((l-1) h, l h)$. By the use of (1.10) we have

Proposition 1 Under the above notations it follows that

(1)) $\left\{\left\|u_{t}^{h}\right\|_{L^{\infty}\left((0, \infty) ; L^{2}(0,1)\right)}\right\}$ is uniformly bounded with respect to $h$

(2)) $\left\{\left\|\bar{u}_{x}^{h}\right\|_{L^{\infty}\left((-h, \infty) ; L^{2}(0,1)\right)}\right\}$ is uniformly bounded with respect to $h$

(3)) $\bar{u}^{h}(t, x) \geq 0$ for each $x$ and $\mathcal{L}^{1}$-a.e. $t$

(4)) $\left\{\left\|u_{x}^{h}\right\|_{L^{\infty}\left((0, \infty) ; L^{2}(0,1)\right)}\right\}$ is uniformly bounded with respect to $h$

(5)) $u^{h}(t, x) \geq 0$ for each $x$ and $\mathcal{L}^{1}$-a.e. $t$

Then there exist a sequence $\left\{h_{j}\right\}$ with $h_{j} \rightarrow 0$ as $j \rightarrow \infty$ and a function $u$ such that

(6)) for any $T>0, u^{h_{j}}$ converges to $u$ as $j \rightarrow \infty$ weakly star in $L^{\infty}\left((0, T) ; L^{2}(0,1)\right)$

(7)) $u_{t}^{h_{j}}$ converges to $u_{t}$ as $j \rightarrow \infty$ weakly star in $L^{\infty}\left((0, \infty) ; L^{2}(0,1)\right)$

(8) $) u_{x}^{h_{j}}$ converges to $u_{x}$ as $j \rightarrow \infty$ weakly star in $L^{\infty}\left((0, \infty) ; L^{2}(0,1)\right)$

(9)) for any $T>0, u^{h_{j}}$ converges to $u$ as $j \rightarrow \infty$ strongly in $L^{\infty}\left((0, T) ; L^{2}(0,1)\right)$

(10)) for any $T>0, \bar{u}^{h_{j}}$ converges to $u$ as $j \rightarrow \infty$ strongly in $L^{\infty}\left((0, T) ; L^{2}(0,1)\right)$

(11)) $s-\lim _{t \searrow t_{0}} u(t)=u_{0}$ in $L^{2}(0,1)$.

This proposition is obtained as a collorary of Proposition 2.2 of [4, Section 2], in which minimizing movement theory for second order hyperbolic equations is developed. Assertions 9), 10) are stronger than corresponding assertions of $[4$, Proposition 2.2]. Since (1.10) implies $\left\{u^{h}(t, \cdot)\right\}$ is contained in a sequentially compact subset of $L^{2}(0,1)$, we can obtain these assertions.

In the terminology of [1] the function $u$ as in Proposition 1 is called a generalized minimizing movement associated with (1.1)-(1.5). Furthermore, if we do not have to subtract a subsequence, $u$ is called a minimizing movement associated with (1.1)-(1.5) (compare to [4]).

Our main theoren is as follows:

Theorem 1.1 The function $u$ as in Proposition 1 is a weak solution to (1.1)(1.5).

This theorem is proved in Section 2. Readers should remark that in general approximation by time semidiscretization method is different from Yosida approximation. A simple example is presented in Section 3.

This work is inspired by [13], in which a problem that describes a movement 
of thin film with an obstacle is investigated. In [13] it is supposed that the film stops when it touches the obstacle and they introduce a different formulation from Schatzman's one. The author expresses his gratitude to Professor Omata, one of the authors of [13], for giving him information about [13].

\section{Proof of Theorem 1.1}

In this section, for the sake of brevity, we write $u^{h_{j}}$ as in Proposition 1 as $u^{h}$. By Proposition 1 we immediately have i) and ii) of the definition of a solution. By the definition of $\mathcal{F}_{l}$ we have $u_{l}-1 \in W_{0}^{1,2}(0,1)$. Thus we have iii) of the definition of a solution by Proposition 18$)$, 9) since $u^{h}-1 \in$ $L^{\infty}\left((0, T) ; W_{0}^{1,2}(0,1)\right)$ for each $h$.

Since $u_{l}$ is the minimizer of $\mathcal{F}_{l}(v)$, we have $\partial \mathcal{F}_{l}\left(u_{l}\right) \ni 0$, namely, for each $\phi \in L^{2}(0,1)$, we have

$$
\int_{0}^{1} \frac{u_{l}-2 u_{l-1}+u_{l-2}}{h^{2}} \phi(x) d x+\int_{0}^{1}\left(u_{l}\right)_{x} \phi_{x} d x+\Phi\left(u_{l}+\phi\right)-\Phi\left(u_{l}\right) \geq 0 .
$$

Then, for each $h$,

$$
\int_{0}^{1} \frac{u_{t}^{h}(t)-u_{t}^{h}(t-h)}{h} \phi(x) d x+\int_{0}^{1} \bar{u}_{x}^{h} \phi_{x} d x+\Phi\left(\bar{u}^{h}(t)+\phi\right)-\Phi\left(\bar{u}^{h}(t)\right) \geq 0(2
$$

for $\mathcal{L}^{1}$-a.e. $t \in(0, \infty)$. Proposition 1 implies $u_{t}^{h}$ and $\bar{u}_{x}^{h}$ converge weakly star to $u_{t}$ and $u_{x}$, respectively, in $L^{\infty}\left((0, \infty) ; L^{2}(0,1)\right)$. Hence we have, for any $\phi \in C_{0}^{1}([0, \infty) \times(0,1))$,

$$
\begin{aligned}
\int_{0}^{\infty} \int_{0}^{1} \frac{u_{t}^{h}(t, x)-u_{t}^{h}(t-h, x)}{h} \phi(t, x) d x d t \\
\\
\quad \longrightarrow-\int_{0}^{\infty} \int_{0}^{1} u_{t} \phi_{t}(t, x) d x d t-\int_{0}^{1} v_{0}(x) \phi(0, x) d x
\end{aligned}
$$

and

$$
\int_{0}^{\infty} \int_{0}^{1} \bar{u}_{x}^{h} \phi_{x} d x d t \longrightarrow \int_{0}^{\infty} \int_{0}^{1} u_{x} \phi_{x} d x d t
$$


(compare to, for example, [2]). Thus, if $\phi \geq 0$, since $\Phi\left(\bar{u}^{h}+\phi\right)=\Phi\left(\bar{u}^{h}\right)=0$, (2.2) implies iv) of the definition of a solution by letting $h \rightarrow 0$.

Finally we prove $\mathrm{v}$ ) of the definition of a solution. First we show, passing to a further subsequence if necessary, $u^{h}$ converges to $u$ uniformly in $(0, T) \times(0,1)$. It is proved by Ascoli-Arzela theorem and hence we should have equicontinuity. Equicontinuity of $x \mapsto u^{h}(t, x)$ is obtained by Proposition 14$)$. In order to have the equicontinuity of $t \mapsto u^{h}(t, x)$ we show the following lemma.

Lemma 2.1 Let $\varphi \in L^{2}(0,1)$ and suppose that $\varphi^{\prime} \in L^{2}(0,1)$ and $\varphi(0)=0$. Then $\|\varphi\|_{L^{\infty}(0,1)} \leq \sqrt{2}\|\varphi\|_{L^{2}(0,1)}^{1 / 2}\left\|\varphi^{\prime}\right\|_{L^{2}(0,1)}^{1 / 2}$.

Proof. Since $\varphi(0)=0$, we have

$$
\varphi(x)^{2}=\varphi(x)^{2}-\varphi(0)^{2}=\int_{0}^{x} \frac{d}{d x}\left(\varphi(y)^{2}\right) d y=\int_{0}^{x} 2 \varphi(y) \varphi^{\prime}(y) d y .
$$

Thus by Schwarz's inequality

$$
\varphi(x)^{2}=2\left|\int_{0}^{x} \varphi(y) \varphi^{\prime}(y) d y\right| \leq 2\|\varphi\|_{L^{2}(0,1)}\left\|\varphi^{\prime}\right\|_{L^{2}(0,1)} .
$$

This implies the conclusion.

Q.E.D.

Since we have

$$
u^{h}(t)-u^{h}(s)=\int_{s}^{t} u_{t}^{h}(\tau) d \tau
$$

for each $t, s \geq 0$, we have

$$
\left\|u^{h}(t)-u^{h}(s)\right\|_{L^{2}(0,1)} \leq\left\|u_{t}^{h}\right\|_{L^{\infty}\left((0, \infty) ; L^{2}(0,1)\right)}|t-s|,
$$

while we have

$$
\left\|u_{x}^{h}(t)-u_{x}^{h}(s)\right\|_{L^{2}(0,1)} \leq 2\left\|u_{x}^{h}\right\|_{L^{\infty}\left((0, \infty) ; L^{2}(0,1)\right)}
$$

By (2.5), (2.6), and Lemma 2.1 we have

$$
\left\|u^{h}(t)-u^{h}(s)\right\|_{L^{\infty}(0,1)} \leq 2\left\|u_{t}^{h}\right\|_{L^{\infty}\left((0, \infty) ; L^{2}(0,1)\right)}^{1 / 2}\left\|u_{x}^{h}\right\|_{L^{\infty}\left((0, \infty) ; L^{2}(0,1)\right)}^{1 / 2}|t-s|^{1 / 2} .
$$

By the use of this inequality 


$$
\begin{aligned}
& \left|u^{h}(t, x)-u^{h}(s, y)\right| \leq\left|u^{h}(t, x)-u^{h}(t, y)\right|+\left|u^{h}(t, y)-u^{h}(s, y)\right| \\
= & \left|\int_{y}^{x} u_{x}^{h}(t, \xi) d \xi\right|+\left|u^{h}(t, y)-u^{h}(s, y)\right| \\
\leq & \left\|u_{x}^{h}\right\|_{L^{\infty}\left((0, T) ; L^{2}(0,1)\right)}|x-y|^{1 / 2}+2\left\|u_{t}^{h}\right\|_{L^{\infty}\left((0, \infty) ; L^{2}(0,1)\right)}^{1 / 2}\left\|u_{x}^{h}\right\|_{L^{\infty}\left((0, \infty) ; L^{2}(0,1)\right)}^{1 / 2}|t-s|^{1 / 2} .
\end{aligned}
$$

By Proposition 11$)$, 4) there exists a constant $C$ independent of $h$ such that

$$
\left|u^{h}(t, x)-u^{h}(s, y)\right| \leq C\left(|x-y|^{1 / 2}+|t-s|^{1 / 2}\right),
$$

namely, $u^{h}$ is equicontinuous in $(0, T) \times(0,1)$ with respect to $h$. Furthermore, letting $s=0$ and $y=0$, we find $\left\{u^{h}\right\}$ is uniformly bounded in $L^{\infty}((0, T) \times$ $(0,1))$. Hereby we have by Ascoli-Arzela theorem that, passing to a further subsequence if necessary, $\left\{u^{h}\right\}$ converges uniformly in $(0, T) \times(0,1)$ to $u$. Let $\phi \in C_{0}^{0}\left([0, T) ; L^{2}(0,1)\right) \cap W_{0}^{1,2}((0, T) \times(0,1))$ satisfy spt $\phi \subset(\{u=0\})^{c}=$ $\{u>0\}$. Here remark that $u$ is continuous with respect to $t$ and $x$. Thus there should be a positive constant $\sigma$ such that $u \geq \sigma$ in spt $\phi$. Without loss of generality we may suppose that $\sup |\phi| \leq \frac{1}{2} \sigma$. Since $u^{h}(t, x)$ converges uniformly to $u(t, x),\left|u(t, x)-u^{h}(t, x)\right|<\frac{1}{2} \sigma$ if $h$ is sufficiently small. Thus we have

$$
u^{h}+\phi=u+\phi+u^{h}-u \geq u-|\phi|-\left|u-u^{h}\right| \geq \sigma-\frac{1}{2} \sigma-\frac{1}{2} \sigma=0 .
$$

Hence $u^{h}+\phi \geq 0$ in $(0, T) \times(0,1)$. Noting that $\bar{u}^{h}(t, x)=u^{h}(l h, x)$ for $(l-1) h<t \leq l h$, we find $\bar{u}^{h}+\phi \geq 0$ in $(0, T) \times(0,1)$. Hence $(2.2)$ implies, for $\mathcal{L}^{1}$-a.e. $t$

$$
\int_{0}^{1} \frac{u_{t}^{h}(t)-u_{t}^{h}(t-h)}{h} \phi(t, x) d x+\int_{0}^{1} \bar{u}_{x}^{h} \phi_{x} d x \geq-\left(\Phi\left(\bar{u}^{h}+\phi\right)-\Phi\left(\bar{u}^{h}\right)\right)=0 .
$$

Replacing $\phi$ with $-\phi$ we have the converse inequality and thus, for $\mathcal{L}^{1}$-a.e. $t$,

$$
\int_{0}^{1} \frac{u_{t}^{h}(t)-u_{t}^{h}(t-h)}{h} \phi(t, x) d x+\int_{0}^{1} \bar{u}_{x}^{h} \phi_{x} d x=0 .
$$

Integrating over $(0, T)$ and letting $h \rightarrow 0$, by (2.3) and (2.4) we have $\mathrm{v})$ of the definition of a solution. 


\section{Appendix}

In this section, giving an example, we assert that approximation by time semidiscretization method is different from Yosida approximation.

Let us define a functional $J: \boldsymbol{R}^{2} \rightarrow[0, \infty]$ by

$$
J(u)=\left\{\begin{array}{l}
\infty \text { if } u^{1}<0 \\
0 \text { if } u^{1} \geq 0
\end{array}\right.
$$

We consider the equation

$$
\left\{\begin{array}{l}
\frac{d^{2} u}{d t^{2}}+\partial J(u) \ni 0 \\
u(t)=u_{0} \\
\frac{d u}{d t}(0)=v_{0}
\end{array}\right.
$$

A weak solution to (3.1) is defined as follows:

Definition 3.1 A function $u:(0, T) \rightarrow \boldsymbol{R}^{2}$ is said to be a weak solution to (3.1) in $(0, T)$ if

i) $u \in\left[W^{1,2}(0, T)\right]^{2}, \quad J(u) \in L^{1}(0, T)$

ii) $\lim _{t \searrow 0} u(t)=u_{0}$ in $\boldsymbol{R}^{2}$

iii) for any $\phi \in\left[C_{0}^{0}[0, T) \cap W^{1,2}(0, T)\right]^{2}$,

$$
\int_{0}^{T}\{J(u+\phi)-J(u)\} d t \geq \int_{0}^{T}\left(u_{t}(t), \phi_{t}(t)\right) d t+\left(v_{0}, \phi\right) .
$$

Now suppose that $u_{0}^{1}>0$ and $v_{0}^{1}<0$. In [9] this equation is solved by Yosida approximation and obtained a solution

$$
u(t)= \begin{cases}u_{0}+t v_{0} & \text { if } t<t^{*} \\ u_{0}+t^{*} v_{0}+\left(t-t^{*}\right) \tilde{v}_{0} & \text { if } t \geq t^{*}\end{cases}
$$


where $t^{*}=\left|v_{0}^{1}\right|^{-1} \operatorname{dist}\left(u_{0},\left\{u^{1}<0\right\}\right)$, namely, the time at which the pass reaches to the interface, and $\tilde{v}_{0}={ }^{t}\left(-v_{0}^{1}, v_{0}^{2}\right)$.

In the sequel we construct a solution to (3.1) in time semidiscretization method, and then it turns out that it is different from (3.2). Letting $u_{0}, v_{0}$ be as in the initial condition and $h$ be a positive number, we construct a sequence $\left\{u_{l}\right\}_{l=-1}^{\infty}$ by setting $u_{-1}=u_{0}-h v_{0}$ for $l=-1$, letting $u_{0}$ be as in the initial condition for $l=0$, and for $l \geq 1$ letting $u_{l}$ as the minimizer of the function

$$
f_{l}(v)=\frac{1}{2 h^{2}}\left|v-2 u_{l-1}+u_{l-2}\right|^{2}+J(v)
$$

in $\boldsymbol{R}^{2}$. Putting $A=\left\{u^{1} \geq 0\right\}$ and $B=\left\{u^{1}<0\right\}$, we have

$$
f_{l}(v)= \begin{cases}\frac{1}{2 h^{2}}\left|v-2 u_{l-1}+u_{l-2}\right|^{2} & \text { if } v \in A \\ \infty & \text { if } v \in B\end{cases}
$$

Thus, if $2 u_{l-1}-u_{l-2} \in A, f_{l}\left(2 u_{l-1}-u_{l-2}\right)=0$ and hence it attaines its minimum, namely $u_{l}=2 u_{l-1}-u_{l-2}$. While, if $2 u_{l-1}-u_{l-2} \in B$,

$$
\inf _{v \in H} f_{l}(v)=\inf _{v \in A} \frac{1}{2 h^{2}}\left|v-2 u_{l-1}+u_{l-2}\right|^{2}
$$

and hence $u_{l}$ is a minimizer of $\left\{\left|v-2 u_{l-1}+u_{l-2}\right| ; v \in A\right\}$. Namely,

$$
\left|u_{l}-2 u_{l-1}+u_{l-2}\right|=\operatorname{dist}\left(2 u_{l-1}-u_{l-2}, A\right) \text {, }
$$

and thus we conclude

$$
u_{l}=\left(\begin{array}{c}
0 \\
2 u_{l-1}^{2}-u_{l-2}^{2}
\end{array}\right) .
$$

In case that $u_{l-2}^{1}>0$ and $u_{l-1}^{1}=0$ we have $2 u_{l-1}^{1}-u_{l-2}^{1}=-u_{l-2}^{1}<0$, namely, $2 u_{l-1}-u_{l-2} \in B$, and in case that $u_{l-2}^{1}=0$ and $u_{l-1}^{1}=0$ we have $2 u_{l-1}^{1}-u_{l-2}^{1}=$ 0 , namely, $2 u_{l-1}-u_{l-2} \in A$. Let $L=\left[t^{*} / h\right]=\max \left\{z \in \boldsymbol{Z} ; z \leq t^{*} / h\right\}$. Then, summing up above, we have

$$
\left\{\begin{array}{l}
u_{l}=u_{0}+l h v_{0} \quad \text { if } 0 \leq l \leq L \\
u_{l}=\left(\begin{array}{c}
0 \\
u_{0}^{2}+l h v_{0}^{2}
\end{array}\right) \text { if } l \geq L+1 .
\end{array}\right.
$$


By the definition of $u^{h}$ we have

$$
u^{h}(t)= \begin{cases}u_{0}+t v_{0} & \text { if } 0 \leq t \leq L h \\
\left(\begin{array}{c}
\left.(L+1)-h^{-1} t\right) u_{0}^{1}+L((L+1) h-t) v_{0}^{1} \\
u_{0}^{2}+t v_{0}^{2}
\end{array}\right) & \text { if } L h<t \leq(L+1) h \\
\left(\begin{array}{c}
0 \\
u_{0}^{2}+t v_{0}^{2}
\end{array}\right) & \text { if }(L+1) h<t .\end{cases}
$$

Now it is easy to check that $u^{h}(t)$ converges to

$$
u(t)= \begin{cases}u_{0}+t v_{0} & \text { if } 0 \leq t \leq t^{*} \\
\left(\begin{array}{c}
0 \\
u_{0}^{2}+t v_{0}^{2}
\end{array}\right) & \text { if } t>t^{*}\end{cases}
$$

strongly in $\left[L^{\infty}(0, T)\right]^{2}$ and that $u$ solves (3.1). However, above $u$ is clearly different from (3.2).

Remark. Here we do not have to substract a subsequence. Hence the function $u$ is a minimizing movement associated with (3.1).

\section{References}

[1] E. De Giorgi, New problems on minimizing movements, Boundary Value Problems for PDE and Applications, Masson, 1993, pp. 81-98.

[2] K. Kikuchi, An analysis of the nonlinear equation of motion of a vibrating membrane in the space of BV functions, J. Math. Soc. Japan 52 (2000), 741766.

[3] _ A remark on Dirichlet boundary condition for the nonlinear equation of motion of a vibrating membrane, Nonlinear Analysis 47 (2001), 1039-1050.

[4] Linear approximation for equations of motion of vibrating membrane with one parameter, J. Math. Soc. Japan 60 (2008), 127-169.

[5] K. Maruo, Existence of solutions of some nonlinear wave equation, Osaka J. Math. 22 (1985), 21-30.

[6] - On certain nonlinear differential equations of second order in time, Osaka J. Math. 23 (1986), 1-53. 
[7] T. Nagasawa, Discrete Morse semiflows and evolution equations, Proceedings of the 16th Young Japanese Mathematicians' Seminar on Evolution Equations, 1994, in Japanese, pp. 1-20.

[8] E. Rothe, Zweidimensionale parabolische randwertaufgaben als grenzfall eindimensionaler randwertaufgaben, Math. Ann. 102 (1930), 650-670.

[9] M. Schatzman, Le système différentiel $\left(d^{2} u / d t^{2}\right)+\partial \phi \ni f$ avec conditions initiales, C. R. Acad. Sc. Paris 284 (1977), A603-A606.

[10] _ A hyperbolic problems of second order with unilateral constraints: the vibrating string with a concave obstacle, J. Math. Anal. Appl. 73 (1980), 138191.

[11] A. Tachikawa, A variational approach to constructing weak solutions of semilinear hyperbolic systems, Adv. Math. Sci. Appl. 3 (1994), 93-103.

[12] _ Weak solution to evolution problems of harmonic maps from noncompact manifolds, Rend. Mat. Appl. (7) 19 (1999), 353-366.

[13] H. Yoshiuchi, S. Omata, K. Svadlenka, and K. Ohara, Numerical solution of film vibration with obstacle, Adv. Math. Sci. Appl. 16 (2006), 33-43. 